\title{
Genetic diversity of environmental Aspergillus flavus strains in the state of São Paulo, Brazil by random amplified polymorphic DNA
}

\author{
Alexandre Lourenço/*/+, Edison Luís Durigon, Paolo Zanotto, \\ Jovita Eugênia Gazzinelli Cruz Madeira***, Adriana Palma De Almeida**, \\ Benedito Correa
}

\begin{abstract}
Instituto de Ciências Biomédicas, Departamento de Microbiologia, Av. Prof. Lineu Prestes 1374, Cidade Universitária, 05508-900
São Paulo, SP, Brasil *Faculdade de Medicina Veterinária, Universidade de Santo Amaro, Universidade Paulista e Faculdades Metropolitanas Unidas, São Paulo, SP, Brasil **Instituto Adolfo Lutz, São Paulo, SP, Brasil ***Fundação Ezequiel Dias, Belo Horizonte, MG, Brasil
\end{abstract}

\begin{abstract}
Aspergillus flavus is a very important toxigenic fungus that produces aflatoxins, a group of extremely toxic substances to man and animals. Toxigenic fungi can grow in feed crops, such as maize, peanuts, and soybeans, being thus of high concern for public health. There are toxigenic and non-toxigenic A. flavus variants, but the necessary conditions for expressing the toxigenic potential are not fully understood. Therefore, we have studied total-DNA polymorphism from toxigenic and non toxigenic A. flavus strains isolated from maize crops and soil at two geographic locations, $300 \mathrm{~km}$ apart, in the Southeast region of Brazil. Total DNA from each A. flavus isolate was extracted and subjected to polymerase chain reaction amplification with five randomic primers through the RAPD (random amplified polymorphic DNA) technique. Phenetic and cladistic analyses of the data, based on bootstrap analyses, led us to conclude that RAPD was not suitable to discriminate toxigenic from non toxigenic strains. But the present results support the use of RAPD for strain characterization, especially for preliminary evaluation over extensive collections.
\end{abstract}

Key words: Aspergillus flavus - random amplified polymorphic DNA - phylogeny genetic diversity

Aflatoxins constitute a group of low molecular weight compounds known to be extremely toxic to animals and man (Bennett \& Klich 2003). They are secondary metabolites produced mainly by Aspergillus flavus and $A$. parasiticus, which are ubiquitous and cosmopolitan fungi able to grow on a wide variety of substrates. However, not all members of each species are toxigenic (Thapar 1988) and, even within potentially toxigenic strains, growth is not necessarily accompanied by toxin production, which depends on favorable environmental conditions such as temperature and humidity (Correa 2000). The economic losses due to fungal contamination and the high costs of control measures are enormous (Bennett \& Keller 1997) and add up to billions of dollars worldwide (Pozzi 2000). For this reason, efforts on scientific research have been towards prevention rather than remedial action (Bennett \& Keller 1997).

The key action for preventing mycotoxins is to block the growth of toxigenic fungi (Fonseca et al. 1974, Bennett $\&$ Keller 1997). But this is not an easy task, since these microorganisms are widely spread through several ecosystems, covering vast geographical areas. Further-

Financial partial support: Direction of Research, Universidade de Santo Amaro

${ }^{+}$Corresponding author: microbiologia @ microbiologia.vet.br Received 22 December 2006

Accepted 1st August 2007 more, they can be easily disseminated by wind and may contaminate food crops at different stages, including harvest, post-harvest, processing, transportation, and even at storage conditions in supermarkets.

Several approaches can be adopted to prevent or minimize food contamination by aflatoxins, such as controlling potential toxigenic-fungi carriers (e.g., insects and rodents), reducing the storage period and controlling humidity and temperature (Correa 2000). The use of antifungal compounds and insecticides, in turn, has been increasingly criticized from the standpoint of environmental concerns, as well as being costly. The production of resistant-plant varieties, although not having yielded strong positive results, is considered to be a promising alternative (Trail et al. 1995) to prevent both A. flavus substrate colonization and aflatoxin production upon fungal proliferation. However, it is important to bear in mind that the host-parasite co-evolution frequently leads to an "arms race" (Ridley 2001), and it is thus unlikely that fungi remain stable with respect to their invasive or toxigenic potential, as they continuously adapt.

The use of non-toxigenic strains for the biological control of toxigenic ones has already been suggested by Egel and collaborators (1994). The non-toxigenic varieties would be artificially disseminated in nature (Egel et al. 1994, Trail et al. 1995) to compete with the toxigenic strains, driving them out of their ecological niches (Trail et al. 1995). In experiments with plants artificially infected with non-toxigenic fugal strains, a reduction by up to $90 \%$ in the aflatoxin contamination was observed (Cotty 1989 apud Tran-Dinh et al. 1999). Yet promising, this result led us to speculate whether this strategy would work with a fungus that is known to undergo high 
levels of genetic recombination (Taylor et al. 1999). As Tran-Dinh and collaborators (1999) have questioned, could the use of a competitive non-toxigenic strain, in the event of genetic recombination, generate a competitive toxigenic strain? To reach a more comprehensive understanding of such matters, population studies of $A$. flavus centered on its genetic variability are indeed required. Knowledge of the relative phylogenetic distances between isolates and strains has launched a debate on the ways by which specific characters are being lost and/ or acquired, as well as on how much these characters are stable (e.g., toxigenic potential). Therefore it will be important to better understand the genetic diversity within this fungal group and the critical factors for retention or loss of characteristics such as toxigenic capacity and virulence to plants.

The use of RAPD (random-amplified polymorphic DNA - Willians et al. 1990) method to determine variability has the advantages of being easy to perform, versatile regarding primers, low-cost and, especially, not requiring previous knowledge on the genetics of the microorganism under study (Willians et al. 1990, DiazGuerra et al. 2000). Besides, with RAPD, the appearance of new ligation sites only creates new annealing sites without impairing pre-existing ones, which would continue to be amplified. Consequently, RAPD allows an independence of characters, which is a clear advantage over techniques that directly or indirectly make use of restriction enzymes, such as restriction fragment length polymorphism (RFLP).

For those reasons, we aimed to compare the genomic profile of toxigenic and non-toxigenic strains isolated from two distinct geographically regions in the state of São Paulo, Brazil (Ribeirão Preto and Capão Bonito) and from different substrates (maize and soil), through RAPD analysis.

\section{MATERIALS AND METHODS}

The strains were isolated from corn crops (Zea mays) and soil in a controlled experiment conducted at two geographically distinct sites, Ribeirão Preto and Capão Bonito (state of São Paulo, Brazil), which are $300 \mathrm{~km}$ apart.

Isolation and identification of fungi strains - From each corn sample, one subsample of $30 \mathrm{~g}$ was taken and then disinfected by immersion in $2 \%$ sodium hypochloride solution for $3 \mathrm{~min}$, followed by 3 rinses with sterile distilled water. From the sub sample, 33 grains were randomly taken and sown on Petri dishes containing agar DRBC (11 kernels per dishes). Incubation was carried out at $25^{\circ} \mathrm{C}$ for 5 days.

Soil samples were analyzed according to the method described by Swanson et al. (1992). For mycoflora isolation, Martin medium was used. The plates was incubated at $25^{\circ} \mathrm{C}$ for 10 days and observed daily.

Mycelial fungi were isolated from corn ears of a hybrid maize, derived from the same set of kernels (according to the methodology proposed by Delp et al. 1986), and from superficial soil samples (0-2 cm depth) around each corn plant at both locations. Colonies whose morphology grossly resembled that of A. flavus (colour of the top surface, and reverse, appearance, topography, and contours) were isolated on Sabouraud dextrose agar and identified according to micromorphological criteria by the microculture technique (Riddel 1950), making use of the shape, colour and size of the phialides, vesicles, conidias and conidiophores (Pitt \& Hocking 1997).

Culture media - Dichloran rose bengal agar with chloramphenicol (Cotty \& Cardwell 1999 - DRBC OXOID) pH 5.6, supplemented with chloramphenicol, was used for the isolation and quantification of fungi on corn kernels.

Martin's medium (Martin 1950) was used for the isolation of soil fungi.

Sabouraud dextrose agar (OXOID) pH5.6, supplemented with chloramphenicol, was used for the identification and maintenance of fungal isolates.

Czapeck medium was used for culturing A. flavus prior to DNA extraction.

Coconut-agar (Lin \& Dianese 1976) was used to test the fungal isolates for aflatoxin production.

Evaluation of toxigenic potential and confirmatory test - The toxigenic potential of the isolates was evaluated according to Lin and Dianese (1976). The procedure used involved extraction with methanol/4\% $\mathrm{KCl}$ (9:1), followed by clarification of the extract with ammonium sulfate and partitioning to chloroform. The content was filtered through filter paper (Whatman no. 1) and evaporate to dryness. Suspended extracts were quantified by thin-layer chromatography (TLC) using aflatoxins standards (Schuller et al. 1973). Confirmation of the identified aflatoxins was carried out derivatization using trifluroacetic acid (Przybylsky 1975). The TLC detection limit was $2 \mu \mathrm{g} / \mathrm{kg}$.

DNA extraction - All fungal isolates were maintained on Sabouraud dextrose agar and further inoculated into flasks containing Czapeck liquid medium prior to totalDNA extraction. After 2-3 days of incubation, the contents of the flasks were aseptically filtered and the mycelial mass was ground with a mortar and pestle in liquid nitrogen. DNA was extracted as described by Innis and White (1990). The amount of DNA (obtained from single extraction) for each sample was estimated by electrophoresis in $1.5 \%$ agarose gels stained with ethidium bromide; DNA concentrations were normalized to about 2.5 ng $\mu \mathrm{l}^{-1}$ by visual comparison with known standards $\left(\right.$ Gibco $\left.^{\circledR}\right)$.

$R A P D P C R$ - Total DNA (10 ng) was submitted to PCR with 5 well established random primers already used with A. flavus (Bayman \& Cotty 1993): P160: CATGGCCACC; P117: TGGCGT CTCCA; P54: GGCGATTTTTGCCG; P10: GCGGCCATAGCAAC; PM1: GCGTAAGCGGGGCATATG. Smaller concentrations of DNA were tested in a pilot experiment, but very low concentrations did not result in a good reproductive technique. The choice of primers currently in use was motivated by our concern to reproduce standard experimental conditions that favor more meaningful comparisons between different works (Welsh \& McClelland 1990). Reaction mixtures were subjected to an incubation step at $94^{\circ} \mathrm{C}$ for $5 \mathrm{~min}$ followed by 45 cycles of $1 \mathrm{~min}$ at 
$94^{\circ} \mathrm{C}, 2 \mathrm{~min}$ at $35^{\circ} \mathrm{C}$, and $3 \mathrm{~min}$ at $72^{\circ} \mathrm{C}$. In the end, samples were kept at $4^{\circ} \mathrm{C}$ until use. The amplification conditions followed a rigorous standardization, including the use of the same Taq-Polimerase $\left(\mathrm{Gibco}^{\circledR}\right)$ and thermocycler (Perkin, model 2.400) sources for all experiments.

In order to confirm that all bands in RAPD reactions were products of the target DNA and not technical artifacts, negative controls with sterile distilled water (Willians et al. 1990, Diaz-Guerra et al. 2000) were introduced instead of DNA samples during extraction with liquid nitrogen. Whenever bands appeared in the negative control (on two occasions very faint bands were detected) the whole run was discarded. Amplifications were carried out in triplicate, which were preferentially run on different days. The amplification products were separated by electrophoresis in $1.5 \%$ agarose $\left(\mathrm{Gibco}^{\circledR}\right)$ gels stained with ethidium bromide (Fig. 1). For each sample, the result selected was the repetition with greatest consistency: clear view of bands and conformity between the repetitions. Very tenuous bands were not considered due to the low stringencies used (Fig. 2).

The results of the band patterns of each gel were noted down in matrices for each primer and after collected together in a single matrix, used for cladistic and phenetic analysis, each one with their own presumptions for the characters in question.

Phenetic analysis - The phenetic analysis was based on the unweighted pair group method using arithmetic averages (UPGMA - Swofford \& Olsen 1990), which

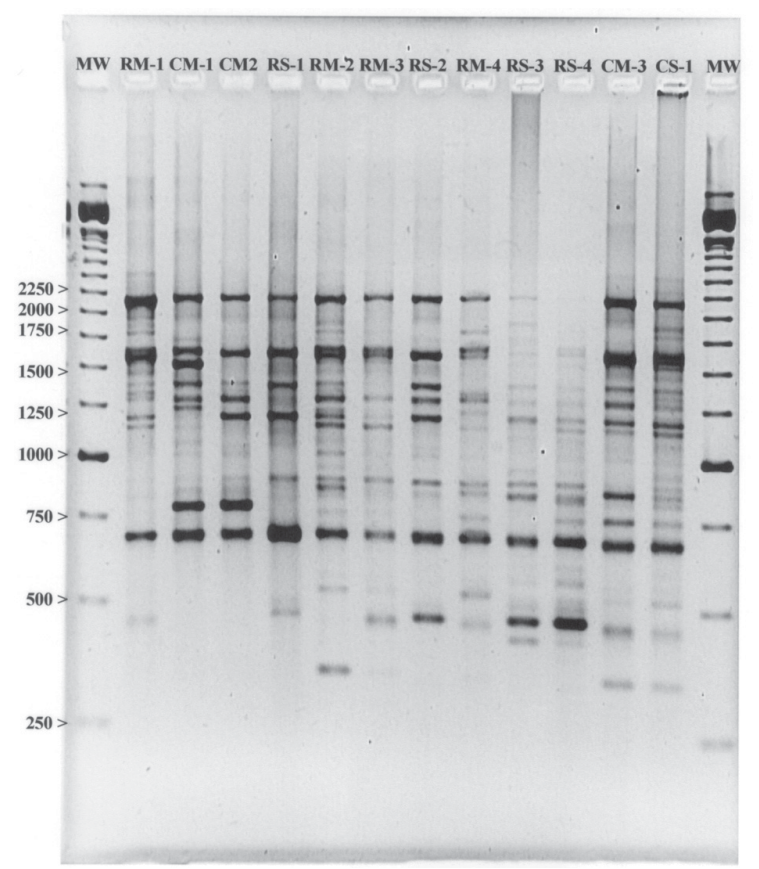

Fig. 1: electrophoretic separation of random amplified polymorphic DNA product of 12 strains from P160 primer after ethidium bromide staining in agar gel $1.5 \%$.

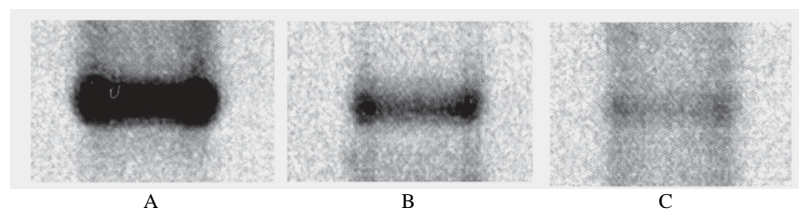

Fig. 2: three examples that represent the intensity of bands after staining with ethidium bromide. In A and B, bands that were considered, and in $\mathrm{C}$ very tenuous band that not considered.

groups by similarities sometimes used in conjunction with RAPD (Bayman \& Cotty 1991) that is part of the PAUP phylogenetic analysis package (Swofford 1998). The adopted assumption for inclusion of characters into the program was: absence of band $=0$; presence of band $=1$.

Cladistic analyses - The cladistic analyses were carried out according two methods based on PARCIMONY by the PAUP Phylogeny program (Berbee et al. 1995). Consensus phylogenetic trees (cladograms) were constructed between the different strains taking into account the characters of the whole set of primers. The basic assumptions (approaches) were: irreversibility of characters (assumption: absence of band $=0$; presence of band $=1$ ) and Dollo (assumption: predominant character $=0$; rare character $=1$ ).

The topology of the trees from these two analyses was compared in order to evaluate the degree of concordance between them.

Reweighting characters was performed until two identical trees were obtained for two consecutive finds (Swofford 1998). This procedure allows getting closer to the best tree, especially for RAPD data.

\section{RESULTS AND DISCUSSION}

Differentiation between toxigenic and non-toxigenic strains - Although only one of our isolates (CM3 - Table I) proved to be toxigenic and, in this respect, this study may not represent a model situation, we have analyzed whether there was a detectable genomic difference between this single strain and the non toxigenic ones. None of the analyses revealed any differentiating pattern (Fig. 3). Similar results have been described in other studies with Aspergillus spp., showing no correlation between DNA band profiles and production or non production of mycotoxyns (Bayman \& Cotty 1993, Croft \& Varga 1994, Jovita \& Bainbridge 1996). Furthermore, Tran-Dinh et al. (1999) conducted a rather thorough investigation on $A$. flavus and $A$. parasiticus and found no relationship between RAPD-based band profile and toxin production. However, on one investigation with isolates of Aspergillus sect. Flavi using RAPD, Egel and collaborators (1994) grouped strains with similar toxigenic capacities, in a more subtle differentiation than the simple classification of toxin producers and non producers. Nonetheless, as the latter researchers employed a different set of primers, any attempt to relate their data to those mentioned before and with our own data would be essentially conjectural. 
Geographic isolation - To wonder what would happen if RAPD was not suitable for characterization, and led to unreliable data, is an interesting speculation. One would expect to form trees by random grouping of samples and that, only eventually, strains from the same origin would group together. However, this was not observed. In fact, the comparison between topologies of the cladistic and phenetic analyses revealed high degree of concordance (Fig. 3). The agreement among different methodologies is one of the indicators of the con-

TABLE I

Aspergillus flavus isolates: abbreviations with respective sites, substrates of origin andtoxigenic potential

\begin{tabular}{lccc}
\hline $\begin{array}{l}\text { Strain } \\
\text { abbreviation }\end{array}$ & Substrate & Region & $\begin{array}{c}\text { Toxigenic } \\
\text { potential }\end{array}$ \\
\hline RM1 & Corn & A & Absent \\
RM2 & Corn & A & Absent \\
RM3 & Corn & A & Absent \\
RM4 & Corn & A & Absent \\
RM5 & Corn & A & Absent \\
RM6 & Corn & A & Absent \\
RS1 & Soil & A & Absent \\
RS2 & Soil & A & Absent \\
RS3 & Soil & A & Absent \\
RS4 & Soil & A & Absent \\
CM1 & Corn & B & Absent \\
CM2 & Corn & B & Absent \\
CM3 & Corn & B & Present \\
CS1 & Soil & B & Absent \\
\hline
\end{tabular}

Region A e B are distant $300 \mathrm{~km}$. sistency of the method adopted (Bayman \& Cotty 1993, Berbee et al. 1995). All in all, it is worth noting that Tran-Dinh and collaborators (1999) have grouped several isolates of A. flavus and A. parasiticus in a clear association with their origins, prompting them to endorse the use of RAPD as a reliable and reproducible methodology with no significant artifacts.

In the present work, we have obtained a non-random array, but one cannot assume that this is a fully discriminatory result and, thus, RAPD usefulness is debatable. Geiser et al. (1998) were unable to find any geographic distribution pattern to work with RFLP. The fact that samples from one region have segregate with samples from another region hampers any attempt to categorically state that there is an association between their origin and their genotyping. This instance may be seen in a cluster gathering 9 samples from region A and two "invaders" (CM3 e CS1) from region B (Fig. 3a). Likewise, there is a clear incursion of the latter samples in the cluster depicted in Fig. 3c. Furthermore sample CM3 appears among those from region A, distant of samples CM1 and CM2 (Fig. 3c). The isolated grouping from region A proceeding from the ground occurred on the three used approaches (Fig. 3) with high Bootstrap value (> 94\%).

In the same way, two samples from region B proceeding from the corn were grouped on the three approaches with a medium Bootstrap value close to $90 \%$. The situation complicates if the Bootstrap values are considered as a whole. If being averagely demanding (> 70\%), only an approach with an expressive number of grouping will be accomplished (Dollo - Table II). In this sense (same table), the phylogenetic analyses had a higher proof than that of the phenetic analyses.

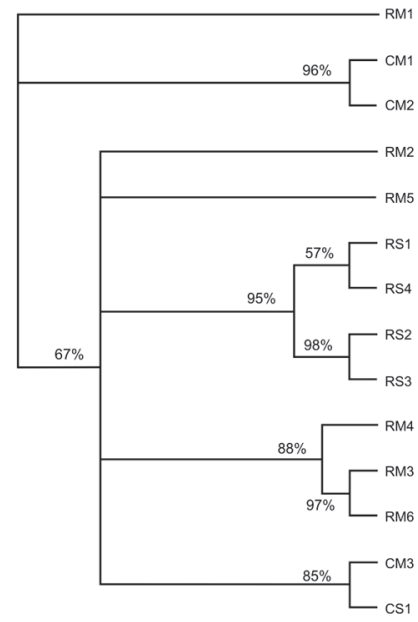

A

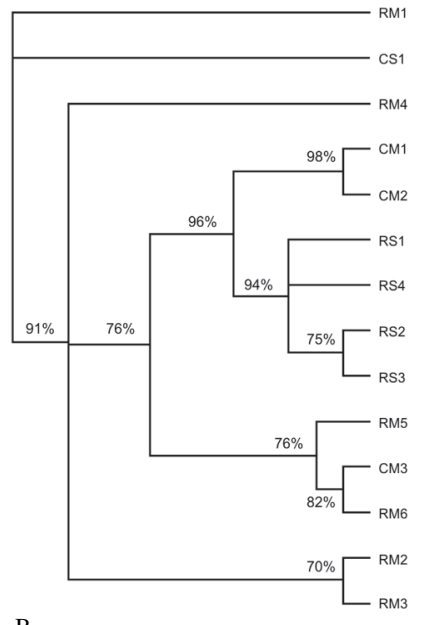

B

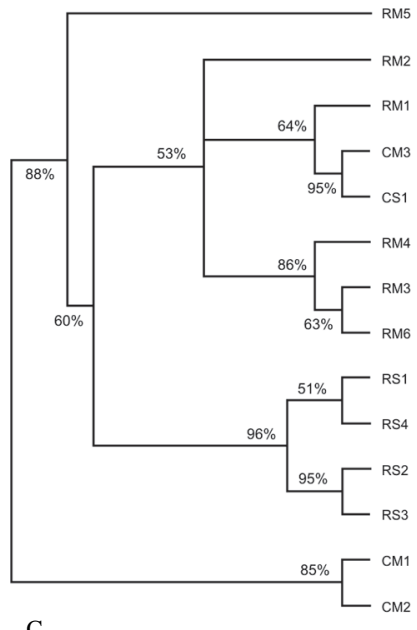

C

Fig. 3: dendrograms constructed from the set of primers. a: obtained by irreversibility approach; b: obtained by Dollo approach; c: obtained by UPGMA approach. Numbers on the branches are bootstrap values and range of 53\% (UPGMA) to 98\% (Dollo and irreversibility). 
TABLE II

Percentual of clustering that reach a Bootstrap value higher than 70 and $90 \%$ in each analysis

\begin{tabular}{lcc}
\hline \multirow{2}{*}{ Analysis } & \multicolumn{2}{c}{$\begin{array}{c}\text { Percentual of clustering that reacha } \\
\text { Bootstrap value higher than }\end{array}$} \\
\hline & $>70 \%$ & $>90 \%$ \\
Dollo & $88.9 \%$ & $44.4 \%$ \\
Irreversibility & $75 \%$ & $50 \%$ \\
UPGMA & $54.5 \%$ & $27.3 \%$ \\
\hline
\end{tabular}

A higher efficiency on the Bootstrap values - which has been the tendency in phylogeny studies (> 90\%) would put the three approaches in an arguable level of validity. However, the concordance of topologies observed can gather important information.

As the A. flavus is known as a species of reproduction exclusively asexual, we can speculate that: (1) given the "mosaic of clones" theory (Kohn 1992), it is possible that species of a region had, over decades, been transported and "invaded" areas with autochthonous clones; (2) if Geiser's et al. speculation (1998) is correct that there is a genetic re-arrangement between the fungus of this species, it is perfectly possible that this generated coinciding patterns of RAPD in different isolated regions (a type of "converging" evolution); and (3) both would be happening to some extent.

This data could be important if we think in terms of a microbiologic control, like what was carried out recently with the malaria mosquito, spreading of the non-toxigenic strain to remove the toxigenic one. Elegant and promising in theory, this approach to controlling micro toxins faces a problem if there or isn't a genetic rearrangement between strains of the Flavi section. This possibility could produce the worst of both worlds, a toxigenic strain even more competitive, removing the others.

Even considering the advantages of being simple, low costing, and applicable to large and genetically unknown collections, these traits, per se, are not enough for electing RAPD as a sole typing methodology. However, the indication of consistence observed show that RAPD-based data could aggregate important information and enrich genotyping studies, specially if they are interpreted in combination with results obtained by other means (Bos \& Stuart 1995).

Of course the importance of RAPD depends on the chosen primers, that can vary in an incredibly wide manner. This makes this technique hostage to a systematic adoption by part of the scientific community, in a "fashion" trend. It is clear that the use of a specific primer is connected to its discriminatory ability, but for the ease of establishing new primers that can eventually be used as standard, we believe that the technique lends itself better to an anarchical diversity than an agreement.

\section{ACKNOWLEDGMENTS}

To Prof. GC Genofre Netto, who trust and give us incentive.

\section{REFERENCES}

Bayman P, Cotty P 1991. Vegetative compatibility and genetic diversity in the Aspergillus flavus population of a single field. Can J Microbiol 69: 1707-1711.

Bayman P, Cotty P 1993. Genetic diversity in Aspergillus flavus: association with aflatoxin production and morphology. Can J Bot 71: 23-31.

Bennett JW, Keller NP 1997. Mycotoxins and their prevention. In T Anke, Fungal Biotechnology, Chapman \& Hall, Weinheim, p. 265-273.

Bennett JW, Klich M 2003. Mycotoxins. Clin Microbiol Rev 16: 497-516.

Berbee ML, Yoshimura A, Taylor JW 1995. Is Penicillium monophiletic? An evaluation of phylogeny in the family Trichocomaceae from 18S, 5,8S and ITS ribossomal DNA sequence data. Mycologia 87: 210-222.

Bos CJ, Stuart K 1995. Genetics of Aspergillus. In The Mycota. Vol. II: Genetics and Biotechnology, Springer-Verlag, Berlin, p. 19-33.

Correa B 2000. Fungos Toxigênicos e Micotoxinas em Milho, do Plantio ao Armazenamento, e os Efeitos da Ação Conjunta de Toxinas em Animais de Laboratório, Thesis, Instituto de Ciências Biomédicas, Universidade de São Paulo, São Paulo, 188 pp.

Cotty PJ 1989. Virulence and cultural characteristics of two Aspergillus flavus strains pathogenic on cotton. Phytopathology 79: 808-814.

Cotty PJ, Cardwell KF 1999. Divergence of west African and North American communities of Aspergillus section flavi. Appl Environ Microbiol 65: 2264-2266.

Croft JH, Varga J 1994. Application of RFLPS in systematics and population genetics of Aspergilli. In The genus Aspergillus, Plenum Press, New York, p. 277-289.

Delp RB, Stwell LJ, Marois JJ 1986. Evaluation of field sampling techniques for estimation of disease incidence. Phytopathology 76: 1299-305.

Diaz-Guerra TM, Mellado E, Cuenca-Estrela M, Gaztelurrutia L, Navarro JIV, Tudela JLR 2000. Genetic similarity among one Aspergillus flavus strain isolated from a patient who underwent heart surgey and two environmental strains obtained from the operating room. J Clin Microbiol 38: 24192422 .

Egel DS, Cotty PJ, Elias KS 1994. Relationships among isolates of Aspergillus sect. Flavi that vary in aflatoxin production. Phytopatology 84: 906-912.

Fonseca H, Martinelli Filho A, Del Nery H, Roncatto E 1974. Espécies de Aspergillus produtoras de aflatoxina, na região araraquense, SP. An Esc Super Agron "Luís De Queiroz" 31: 519-536.

Geiser DM, Dorner JW, Horn BW, Taylor JW 2000. The phylogenetics of mycotoxin and sclerotium production in $\mathrm{As}$ pergillus flavus and Aspergillus oryzae. Fungal Genet Biol 31: 169-179.

Geiser DM, Oitt JI, Taylor JW 1998. Cryptic speciation and recombination in the aflatoxin-producing fungus Aspergillus flavus. Proc Natl Acad Sci USA 95: 388-393.

Innis MA, White TJ 1990. PCR Protocols: A Guide to Meth- 
ods and Aplications, Academic Press Inc., New York, p. 282-287.

Jovita JG, Bainbridge BW 1996. Identification of Aspergillus flavus, Aspergillus oryzae and Aspergillus parasiticus using randomly amplified polymorphic DNA (RAPD) markers. In International IUPAC Symposium on Mycotoxins and Phycotoxins, Roma, p. 292.

Kohn, LM 1992. Developing new characters for fungal systematics: an experimental approach for determining the rank of resolution. Mycologia 84: 139-53.

Lin MT, Dianese JC 1976. A coconut-agar medium for rapid detection of aflatoxin production by Aspergillus spp. Phytopathology 66: 1466-1469.

Martin JP 1950. Use of acid rose bengal and streptomycin in the plate method for estimating soil fungi. Soil Sci 69: 215-232.

Pitt JI, Hocking AD 1997. Fungi and Food Spoilage, Chapman \& Hall, London, 593 pp.

Pozzi CR 2000. Efeitos da Administração Oral Prolongada de Fumonisina B1 e Aflatoxina Bl em Ratos (Rattus norvegicus), $\mathrm{PhD}$ Thesis, Instituto de Ciências Biomédicas, Universidade de São Paulo, São Paulo, 179 pp.

Przybylsky W 1975. Formation of aflatoxin derivatives on thin layer chromatographoplates. J Assoc Off Anal Chem 58: 163-165.

Riddel RW 1950. Permanent stained mycological preparations obtained by slid culture. Mycologia 42: 265-270.

Ridley M 2001. Genoma, Record, Rio de Janeiro, 398 pp.

Schuller PL, Vverhulsoonk CAH, Paulsch WE 1973. Analysis of aflatoxin $\mathrm{M}_{1}$ in liquid and powdwred milk. Pure Appl Chem 35: 291-296.

Swanson KM, Busta FF, Petterson EH, Johnson MG 1992. Colony count methods. In C Vanxderzant, DS Splittoesser, Compendium of Methods for the Microbiological Examination of Foods, American Public Health Association, New York.

Swofford DL 1998. PAULP*: Phylogenetic analysis using parsimony (*and other methods). version 4, Sinauer Associates, Massachusetts.

Swofford DL, Olson GJ 1990. Phylogeny reconstruction. In DM Hillis, C Moritz Molecular Systematics, Sinauer Associates Inc., New York, 515 pp.

Taylor JW, Geiser DM, Burtin A, Koufopanou V 1999. The evolutionary biology and population genetics underlying fungal strain typing. Clin Microbiol Rev 12: 126-146.

Thapar GS 1988. Metabolic behaviour of aflatoxin producing strain and non-toxigenic strain of Aspergillus flavus to different sources of nitrogen and glucose concentration. Mycopathologia 102: 9-12.

Trail F, Mahanti N, Linz J 1995. Molecular biology of aflatoxin biossynthesis. Microbiology 141: 755-765.

Tran-Dinh N, Pitt JI, Carter DA 1999. Molecular genotype analysis of natural toxigenic and nontoxigenic isolates of Aspergillus flavus and Aspergillus parasiticus. Mycol Res 103: 14851490.

Welsh J, McCleland M 1990. Fingerprint genomes using PCR with arbitrary iniciadores. Nucleic Acid Research 18: 7213-7218.

Willians JGK, Kubelik AR, Livak KJ, Rafalski JA, Tney SV 1990. DNA polymorphisms amplified by arbitrary iniciadores are useful as genetic markers. Nucleic Acid Res 18: 6531-6535. 